Walter Varillas 1

\footnotetext{
1 Red del Trabajo Infantil Peligroso, Organización Internacional del Trabajo, Programa de Erradicación del Trabajo Infantil

(Red/ TIP - IPEC/OIT). Las Flores 271, San Isidro, Lima, Peru.

wvarillas@amauta.rcp.net.pe
}

\section{La red sobre trabajo infantil peligroso (Red Tip)}

\author{
The hazardous child labor network (Red Tip)
}

Abstract In the world, approximately 351.7 millions children between 5 and 17 years old work. Of them, 170,5 millions (48.5\%) work at the hazardous child labor forms. A high percentage is in agriculture, others in mines, manufactures, brick makers, predominantly in informal economy. The 138 Convention of ILO and the 182 Convention, define as hazardous child labor activities that can affect the health, safety and morality of the children. Studies on the children at work point out their particular susceptibility to some occupational risks, increasing the danger for their normal development and growth. "They are not little adults". The occupational health professionals, can collaborate with the professionals and the organizations specialized on child labor in the definition and characterization of hazardous child labor. The $\mathrm{N}$ etwork about $\mathrm{H}$ azardous Child Labor (Red TIP) aims to facilitate the collaboration between professionals and organizations, focusing on the elimination of the hazardous child labor, in the rescuing of the young workers and on the development of their opportunity to smile now and in the future. $\mathrm{K}$ ey words $\mathrm{H}$ azardous child labor, OIT conventions, W orst forms of the child labor
Resumen En el mundo, aproximadamente 351.7 millones de niños entre 5 y 17 años realizaban algún tipo de actividad económica, de ellos 170.5 millones (48.5\%) realizaban algún tipo de trabajo considerado peligroso. U $\mathrm{n}$ alto porcentaje se encuentra en la agricultura, otros en minas, manufacturas, ladrilleras, predominantemente en la economía informal. El Convenio 138 (cobrela edad mínima de admisión en el empleo) dela OIT y el Convenio 182 (sobre las peores formas de trabajo infantil), definen como trabajo infantil peligroso el que puede afectar la salud, seguridad y moralidad de los menores. Estudios específicos sobre los menores muestran su susceptibilidad particular frente a los riesgos laborales, aumentando la peligrosidad para su normal desarrollo y crecimiento: "Ios niños no son adultos pequeños". Los profesionales de la seguridad y salud en el trabajo pueden colaborar con los profesionales y las organizaciones especializadas en el trabajo infantil, en la definición y caracterización de lo que significa el trabajo infantil peligroso. Para ello se ha conformado la Red sobre Trabajo Infantil Peligroso (Red TIP), con la finalidad de articular estos dos espacios, orientados a eliminar el trabajo infantil peligroso y rescatar al menor y devolverle la oportunidad de sonreír ahora y en el futuro.

Palabras-clave Trabajo infantil peligroso, Convenios OIT, Peores formas de trabajo infantil, $\mathrm{N}$ iños trabajadores 


\section{La magnitud del trabajo infantil peligroso}

De acuerdo al Informe Global "Un futuro sin trabajo infantil", publicado por la OIT en mayo del 2002, para el año 2000 aproximadamente 351.7 millones de niños entre 5 y 17 años realizaban algún tipo de actividad económica. De este grupo, 170.5 millones (48.5\%) realizaban algún tipo de trabajo considerado peligroso (Cuadro 1). La OIT define como trabajo infantil peligroso aquella actividad desarrollada por las personas menores de 18 años y que por su naturaleza o por las condiciones en que se lleva a cabo, es probable que dañe la salud, la seguridad o la moralidad de los niños.

Las cifras de este informe muestran la magnitud del problema del trabajo infantil peligroso, que representa el $11 \%$ del total de la población infantil comprendida entre los 5 y los 17 años a nivel mundial. De manera gráfica podríamos decir que de cada 10 niños que hay en el mundo 2 realizan actividades económicas y uno de ellos lo hace en trabajos peligrosos. También podría decirse que de cada dos niños que realizan actividades económicas, uno de ellos labora en trabajos peligrosos.

En el caso de los llamados países en desarrollo, el trabajo infantil se da principal mente en la actividad agrícola rural, de manera secundaria en la manufactura, comercio, servicios, particularmente en las formas de economía informal. Es mayor el trabajo de los niños que el de las niñas, conforme avanza la edad (Cuadro 2).

\section{Prestar atención al trabajo infantil peligroso}

El trabajo infantil peligroso no sólo es un problema relevante por su magnitud, sino además por su gravedad. El citado Informe Global hace referencia a la necesidad de intervenciones de largo plazo para la reducción de la pobreza y para promover un crecimiento económico sostenido, además de intervenciones en los mis-

\section{Cuadro 1}

Niños de 5 a 17 años que realizan actividades económicas y trabajos peligrosos en el mundo. Por grupos de edad.

\begin{tabular}{|c|c|c|c|}
\hline & De 5 a 14 años & De 15 a 17 años & Total \\
\hline Niños económicamente activos & $\begin{array}{c}210.800 .000 \\
(100.0 \%)\end{array}$ & $\begin{array}{c}140.900 .000 \\
(100.0 \%)\end{array}$ & $\begin{array}{c}351.700 .000 \\
(100.0 \%)\end{array}$ \\
\hline Niños que realizan trabajos peligrosos & $\begin{array}{c}111.300 .000 \\
(52.8 \%)\end{array}$ & $\begin{array}{c}59.200 .000 \\
(42 \%)\end{array}$ & $\begin{array}{c}170.500 .000 \\
(48.5 \%)\end{array}$ \\
\hline
\end{tabular}

Elaboración en base a: OIT (2002), p. 20.

\section{Cuadro 2}

Estimado de la distribución del trabajo infantil en los países en desarrollo por actividad económica.

\begin{tabular}{|c|c|c|c|}
\hline Actividades económicas & $\underset{\%}{\text { Niños }}$ & $\underset{\%}{\text { Niñas }}$ & $\begin{array}{c}\text { Total } \\
\%\end{array}$ \\
\hline Agricultura, caza, silvicultura, pesca & 68.8 & 75.3 & 70.4 \\
\hline Manufactura & 9.4 & 7.9 & 8.3 \\
\hline Comercio & 10.4 & 5.1 & 8.3 \\
\hline Servicios comunitarios, sociales y personales & 4.7 & 8.9 & 6.5 \\
\hline Transporte, almacenamiento, comunicaciones & 3.8 & - & 3.8 \\
\hline Construcción & 2.0 & 1.9 & 1.9 \\
\hline M inas y canteras & 1.0 & 0.9 & 0.8 \\
\hline Total & 100.0 & 100.0 & 100.0 \\
\hline
\end{tabular}

Elaboración en base a: OIT (2002), p. 25. 
mos lugares donde se origina el problema, donde está la pobreza que crea las peores formas de trabajo infantil y donde las peores formas de trabajo infantil dan origen a la pobreza.

A pesar de los avances realizados resulta difícil definir el trabajo infantil peligroso como una categoría específica de trabajo dentro del grupo de las peores formas de trabajo infantil. Al respecto, el informe de la OIT señala lo siguiente:

Así pues, no siempre es fácil trazar los límites del trabajo peligroso, sobre todo cuando el daño que se está haciendo a los niños no es perceptible a corto plazo. El trabajo peligroso está ya señalado en el Convenio N ro. 138, como el trabajo que requería una edad mínima para la admisión de 18 años... Su identificación como una delas peores formas de trabajo infantil da una nueva urgencia a la acción dirigida a eliminarlo (OIT, 2002).

En este documento se menciona que el trabajo puede perjudicar al niño por la tarea en sí que hay que realizar, por los instrumentos que se utilizan, por los horarios o condiciones de trabajo. También puede haber otros factores que afecten el desarrollo físico, mental, emocional, psicológico, moral o espiritual de los menores.

Los menores de 18 años corren riesgos de salud y seguridad superiores a los de los adultos; por su proceso de crecimiento y desarrollo, son más susceptibles a los riesgos laborales y pueden ser afectados de manera irreversible, como sustenta Forastieri (1997). Tensiones físicas crónicas sobre huesos y articulaciones en cre cimiento pueden impedir el desarrollo de los mismos, causar lesiones medulares y otras deformaciones definitivas (OIT, 2002). Estos riesgos son acentuados por el estado de desnutrición, agotamiento precoz, menor madurez que el adulto, máquinas y herramientas no adaptadas a sus características. M uchos riesgos no son observables a simple vista por sus efectos tardíos, como el caso de los efectos nocivos de los plaguicidas o el golpe de calor en la agricultura.

La OIT reconoce la necesidad de aprender más sobre los efectos a corto y largo plazo de los distintos tipos de trabajo de niñas y niños de diversas edades y estados de salud. Es necesario adquirir esos conocimientos para poder de cidir quétipos de trabajo han de prohibirse a los niños menores de 18 años y para planificar la adecuada rehabilitación de los niños que hayan sido retirados de trabajos peligrosos. Pese a no haber datos completos sobre las lesiones y en- fermedades que causa el trabajo infantil, podemos observar al gunas cifras (OIT, 2002):

- En Estados U nidos, la tasa de lesiones por hora de trabajo en el caso de los niños y adolescentes casi duplica a la de los adultos. En el período 1992-1998, la tasa de mortalidad de trabajadores jóvenes alcanzó su máximo en la agricultura, la silvicultura y la pesca, seguidas por el comercio al detalle y la construcción.

- En una encuesta realizada en Dinamarca, Finlandia, N oruega y Suecia en 1997-1998, se observaron tasas de lesiones entre el 3 y 9 por ciento en los niños que trabajan antes o después de la escuela. En Dinamarca se observaron mayores tasas de accidentes de niños en la agricultura, que en otros sectores.

- En un estudio de OIT realizado en 1997 en algunos países en desarrollo, se observaban las siguientes tasas medias de enfermedades y lesiones entre niños: $25.6 \%$ en la construcción; $18.1 \%$ en transporte, almacenamiento, comunicaciones; $15.9 \%$ en minería y cantería, siendo notable que las tasas sean mayores en niñas, con excepción de transporte.

\section{Algunas explicaciones sobre el mayor riesgo de los menores a accidentes y enfermedades ocupacionales}

Forastieri (1997) y Hiba (2002) sistematizan las siguientes condiciones particulares de susceptibilidad frente a los riesgos de los niños respecto a los adultos: inmadurez de órganos y tejidos, más alto consumo metabólico y de oxígeno, mayores necesidades de energía, resistencia física más baja, resistencia más baja a cambios de temperatura, menor destreza manual para operar herramientas, alta capacidad de absorción, alta vulnerabilidad psicológica.

M encionan que esto puede ser agravado por efectos a largo plazo de la desnutrición, enfermedades del trabajo y contagiosas adquiridas por los niños en trabajos peligrosos, con las siguientes consecuencias: fatiga crónica, agotamiento físico y estrés mental, reducción de la capacidad física para trabajar en la adultez, crecimiento retardado, capacidad auditiva dañada, deterioro neurológico, daños y discapacidades.

Agregan algunas condiciones que agravan la situación del trabajo infantil como: la deficiente información sobre los riesgos en el trabajo, la inexperiencia laboral y la deficiente información laboral, los menores no conocen los riesgos, o conocen menos que los adultos, por 
lo que se exponen más, por lo general no están entrenados para el trabajo que van a desarroIlar, ni para tomar medidas de protección, estando expuestos de manera abierta y directa. No existen equipos de protección personal para menores, los que existen han sido fabricados para adultos. Discrepancias entre las tareas indicadas y las tareas realizadas. Las tareas, las herramientas, equipos y máquinas, son hechas para adultos, no están diseñadas ni pensadas para los menores. Exposición a agentes físicosy biológicos peligrosos. Exposición a productos químicos tóxicos. Ambiente psíquico y social inadecuado. Condiciones higiénicas pobres. Limitado acceso a los servicios médicos. Desgaste e incapacidad prematura, lesiones corporales y accidentes mortales. Los niños son sensibles a seguir actitudes, atavismos o comportamientos de riesgo de los adultos. Los menores desean "destacar", para demostrar que son "iguales" o capaces como los adultos, no midiendo el mayor riesgo que podría causarles esta actitud.

\section{Características generales del problema del trabajo infantil en América Latina}

De acuerdo al IPEC-OIT y a pesar de la carencia de estudios fiables, se puede cifrar en 7.6 millones de niños de entre diez y catorce años que trabajan en América Latina. Aunque si se incluyen las tareas domésticas, a los menores de diez años y las propias subestimaciones estadísticas, la cifra total de niños trabajadores se situaría entre dieciocho y veinte millones. Esto significa que uno de cada cinco niños está económicamente activo en América Latina.

O tros aspectos significativos son la mayor participación de niños (60\%) que de niñas (40\%) y una mayor presencia en el ámbito rural (55\%) que en el urbano (45\%). La mayoría (el $90 \%$ ) trabaja en el sector informal frente a un $10 \%$ que trabaja en el sector estructurado de la economía.

La proporción de asalariados llega a representar entre el $60-70 \%$ en las áreas urbanas y alrededor del $50 \%$ en el conjunto total de los niños trabajadores.

Las jornadas de trabajo, en la mayoría de los casos son superiores a los límites máximos establecidos en las legislaciones. La media es de 45 horas semanales y aun los que van a la escuela, dedican 35 horas a la semana a diversas ocupaciones laborales. Los ingresos son tam- bién muy bajos, recibiendo ingresos menores a los adultos por trabajos similares.

\section{Características generales del trabajo infantil peligroso en América del Sur}

Los niños que trabajan están expuestos a sufrir lesiones y enfermedades en una proporción tal que resulta muy preocupante. Por tanto, al establecer los peligros del trabajo infantil es necesario traspasar el relativamente limitado concepto de "riesgo laboral" tal como se aplica a los adultos y ampliarlo para que abarque también el desarrollo infantil. Si los niños trabajadores en general son vulnerables a los riesgos relacionados con el trabajo, los niños y las niñas muy pequeños lo son todavía más. Sumado a lo anterior las jornadas de trabajo, en la mayoría de los casos, son superiores a las legislaciones nacionales.

Se ha verificado en diferentes países un al to nivel de ocupación infantil en ladrilleras, minas, canteras de piedra, mercados, coheterías, servicio doméstico o el sector agrícola entre otros. Los riesgos y perjuicios físicos para estos menores son evidentes: inhalaciones tóxicas, quemaduras, pérdida parcial de vista, mutilaciones, afecciones broncopulmonares, reacciones alérgicas, problemas dermatológicos 0 enfermedades infecto contagiosas (Cuadro 3).

\section{La respuesta de los países y de la O IT}

Frente a este problema y, en general, al problema del trabajo infantil, los países, representados en la OIT, como organismo de $\mathrm{N}$ aciones Unidas de carácter tripartito, han adoptado una serie de convenios que buscan crear conciencia sobre la gravedad y magnitud del problema, sobre la necesidad de adoptar políticas y acciones efectivas para evitar situaciones que puedan afectar la integridad física o moral de la población infantil trabajadora, que puedan impedir sus posibilidades de desarrollo.

Así, tenemos el Convenio 138 (sobre la edad mínima para el empleo, 1973), OIT (1973), y el Convenio 182 (sobre las peores formas de trabajo infantil, 1999), OIT (1999). Ambos convenios han sido ratificados por unos 120 países alrededor del mundo (Cuadro 4). 
Cuadro 3

Sectores de alto riesgo identificadas por IPEC por país.

\begin{tabular}{|c|c|}
\hline Argentina & Ladrilleras, M ercados, Industria del cuero, Agricultura, Fabricación de Helados \\
\hline Bolivia & M inería, Zafra, Construcción, Trabajo callejero, Agricultura \\
\hline Brasil & Hornos de carbón, Pedreras, Preparación del sisal, Depósitos de basura \\
\hline Chile & M inería, Agricultura, Trabajo callejero \\
\hline Colombia & M inería, Agricultura \\
\hline Costa Rica & $\begin{array}{l}\text { Servicio doméstico, Construcción, Prostitución, Banano, M aquila, } \\
\text { Procesamiento de mariscos }\end{array}$ \\
\hline Ecuador & Floricultura, Trabajo callejero, Construcción \\
\hline El Salvador & $\begin{array}{l}\text { Curiles, M aquila, Pirotecnia, Construcción, Cafetales, Prostitución, } \\
\text { Trabajo callejero, Basura }\end{array}$ \\
\hline Guatemala & $\begin{array}{l}\text { Sector de la cal, Cafetales, M inería, Pirotecnia, Servicio doméstico, M aquila, } \\
\text { Construcción, Transporte, Basura }\end{array}$ \\
\hline Honduras & $\begin{array}{l}\text { Industria del cuero, Panadería, M aquilas, M aderas, M etalurgia, Construcción, } \\
\text { Ejército, Industria fármacos, Industria química, Industria en general }\end{array}$ \\
\hline M éxico & Cafés y bares, Talleres mecánicos, Ladrilleras, Agricultura \\
\hline Nicaragua & Cafetales, Banano, Arroz, Tabaco, Algodón, Ganadería, Trabajo callejero \\
\hline Panamá & Trabajo callejero, Servicio doméstico, Zafra, Carga \\
\hline Paraguay & Trabajo callejero, Servicios domésticos \\
\hline Perú & $\begin{array}{l}\text { Lavaderos de oro, Ladrilleras, Picapedreros, Camales, Construcción, M etalurgia, } \\
\text { Procesami ento hoja de coca, Pirotecnia, Basura, M inería }\end{array}$ \\
\hline $\begin{array}{l}\text { República } \\
\text { Dominicana }\end{array}$ & Agricultura, Servicio doméstico, Basura, Prostitución \\
\hline
\end{tabular}

\section{El Convenio 138 establece la edad mínima de admisión al empleo}

El Convenio 138 trata sobre la edad mínima de admisión al empleo, estableciendo el compromiso de los Estados que ratifiquen esta norma internacional, de seguir una política nacional de abolición del trabajo infantil y de elevación progresiva de la edad mínima de admisión al empleo 0 al trabajo a un nivel que haga posible el más completo desarrollo físico y mental de los menores (artículo 1).

En su artículo 3, párrafo 1, alude a la prohibición del trabajo infantil peligroso, al decir: La edad mínima de admisión a todo tipo de empleo o trabajo que por su naturaleza o las condiciones en que se realice pueda resultar peligroso para la salud, la seguridad o la moralidad de los menores no deberá ser inferior a dieciocho años.
El Artículo 5, párrafo 3, indica las actividades económicas en las que no debe realizarse formas de trabajo infantil, principalmente las orientadas al comercio.

\section{El Convenio 182 sobre la prohibición de las peores formas de trabajo infantil y la acción inmediata para su eliminación}

El Convenio 182 de la OIT establece la adopción de medidas inmediatas y eficaces para conseguir la prohibición y la eliminación de las peores formas de trabajo infantil (artículo 1). Señala (artículo 3) que la expresión las peores formas de trabajo infantil abarca:

a) todas las formas de esclavitud o las prácticas análogas a la esclavitud, como la venta y el tráfico de niños, la servidumbre por deudas y la 


\section{Cuadro 4}

Estado actual de la ratificación de los convenios 138 - sobre edad mínima de admisión al empleo, y 182 - sobre peores formas de trabajo infantil en América Latina y El Caribe.

\begin{tabular}{|c|c|c|}
\hline País & $\begin{array}{l}\text { Convenio } \\
138\end{array}$ & $\begin{array}{l}\text { Convenio } \\
182\end{array}$ \\
\hline Antigua y Barbuda & $\begin{array}{l}\text { Fecha de ratificación: } 17.3 .1983 \\
\text { Edad mínima especificada: } 16 \text { años }\end{array}$ & Fecha de ratificación: 16.9.2002 \\
\hline Argentina & $\begin{array}{l}\text { Fecha de ratificación: } 11.11 .1996 \\
\text { Edad mínima especificada: } 14 \text { años }\end{array}$ & Fecha de ratificación: 5.2.2001 \\
\hline Bahamas & $\begin{array}{l}\text { Fecha de ratificación: } 31.10 .2001 \\
\text { Edad mínima especificada: } 14 \text { años }\end{array}$ & Fecha de ratificación: 14.6 .2001 \\
\hline Barbados & $\begin{array}{l}\text { Fecha de ratificación: } 4.1 .2000 \\
\text { Edad mínima especificada: } 15 \text { años }\end{array}$ & Fecha de ratificación: 23.10 .2000 \\
\hline Belice & $\begin{array}{l}\text { Fecha de ratificación: } 6.3 .2000 \\
\text { Edad mínima especificada: } 14 \text { años }\end{array}$ & Fecha de ratificación: 6.3 .2000 \\
\hline Bolivia & $\begin{array}{l}\text { Fecha de ratificación: } 11.6 .1997 \\
\text { Edad mínima especificada: } 14 \text { años }\end{array}$ & Fecha de ratificación: 6.6.2003 \\
\hline Brasil & $\begin{array}{l}\text { Fecha de ratificación: } 28.6 .2001 \\
\text { Edad mínima especificada: } 16 \text { años }\end{array}$ & Fecha de ratificación: 2.2.2000 \\
\hline Chile & $\begin{array}{l}\text { Fecha de ratificación: } 1.2 .1999 \\
\text { Edad mínima especificada: } 15 \text { años }\end{array}$ & Fecha de ratificación: 17.7.2000 \\
\hline Colombia & $\begin{array}{l}\text { Fecha de ratificación: } 2.2 .2001 \\
\text { Edad mínima especificada: } 14 \text { años }\end{array}$ & $\begin{array}{l}\text { Fecha de ratificación: } 27.11 .2001 \\
\text { (Pendiente depósito en Ginebra) }\end{array}$ \\
\hline Costa Rica & $\begin{array}{l}\text { Fecha de ratificación: } 11.6 .1976 \\
\text { Edad mínima especificada: } 15 \text { años }\end{array}$ & Fecha de ratificación: 10.9.2001 \\
\hline Cuba & $\begin{array}{l}\text { Fecha de ratificación: } 7.3 .1975 \\
\text { Edad mínima especificada: } 15 \text { años }\end{array}$ & \\
\hline Dominica & $\begin{array}{l}\text { Fecha de ratificación: } 27.9 .1983 \\
\text { Edad mínima especificada: } 15 \text { años }\end{array}$ & Fecha de ratificación: 4.1.2001 \\
\hline Ecuador & $\begin{array}{l}\text { Fecha de ratificación: } 19.9 .2000 \\
\text { Edad mínima especificada: } 14 \text { años }\end{array}$ & Fecha de ratificación: 19.9.2000 \\
\hline El Salvador & $\begin{array}{l}\text { Fecha de ratificación: } 23.1 .1996 \\
\text { Edad mínima especificada: } 14 \text { años }\end{array}$ & Fecha de ratificación: 12.10 .2000 \\
\hline Guatemala & $\begin{array}{l}\text { Fecha de ratificación: } 27.4 .1990 \\
\text { Edad mínima especificada: } 14 \text { años }\end{array}$ & Fecha de ratificación: 11.10 .2001 \\
\hline Guyana & $\begin{array}{l}\text { Fecha de ratificación: } 15.4 .1998 \\
\text { Edad mínima especificada: } 15 \text { años }\end{array}$ & Fecha de ratificación: 15.1.2001 \\
\hline Honduras & $\begin{array}{l}\text { Fecha de ratificación: } 9.06 .1980 \\
\text { Edad mínima especificada: } 14 \text { años }\end{array}$ & Fecha de ratificación: 25.10 .2001 \\
\hline M éxico & & Fecha de ratificación: 30.6 .2000 \\
\hline Nicaragua & $\begin{array}{l}\text { Fecha de ratificación: } 2.11 .1981 \\
\text { Edad mínima especificada: } 14 \text { años }\end{array}$ & Fecha de ratificación: 6.11 .2000 \\
\hline Panamá & $\begin{array}{l}\text { Fecha de ratificación: } 31.10 .2000 \\
\text { Edad mínima especificada: } 14 \text { años }\end{array}$ & Fecha de ratificación: 31.10 .2000 \\
\hline
\end{tabular}

(continua) 
Cuadro 4 (continuación)

\begin{tabular}{lll}
\hline País & $\begin{array}{l}\text { Convenio } \\
138\end{array}$ & $\begin{array}{l}\text { Convenio } \\
182\end{array}$ \\
\hline Paraguay & & Fecha de ratificación: 7.3 .2001 \\
Perú & $\begin{array}{l}\text { Ratificado por el país el 13.11.2002 } \\
\text { Edad mínima especificada: } 14 \text { años }\end{array}$ & Fecha de ratificación: 10.1 .2002 \\
República Dominicana & Fecha de ratificación: 15.6 .1999 & Fecha de ratificación: 15.11 .2000 \\
Saint Kitts \& Nevis & Edad mínima especificada: 14 años & Fecha de ratificación: 12.10 .2000 \\
Santa Lucia & & Fecha de ratificación: 6.12 .2000 \\
San Vincentey Las Granadinas & & Fecha de ratificación: 4.12 .2001 \\
U ruguay & & Fecha de ratificación: 3.8 .2001 \\
Venezuela & Fecha de ratificación: 2.6 .1977 & \\
\hline
\end{tabular}

Información actualizada a julio 2003.

Fuentes de los datos: ILOLEX-OIT

Elaboración de la información: Equipo IPEC Sudamérica

Convenio 138: 23 países

Convenio 182: 26 países (Colombia pendiente de Depósito)

condición de siervo, y el trabajo forzoso u obligatorio, incluido el reclutamiento forzoso u obligatorio de niños para utilizarlos en conflictos armados;

b) la utilización, el reclutamiento o la oferta de niños para la prostitución, la producción de pornografía 0 actuaciones pornográficas;

c) la utilización, el reclutamiento o la oferta de niños para la realización de actividades ilícitas, en particular la producción y el tráfico de estupefacientes, tal como se definen en los tratados internacionales pertinentes, $y$

d) el trabajo que, por su naturaleza o por las condiciones en que se lleva a cabo, es probable que dañe la salud, la seguridad o la moralidad de los niños.

Es este grupo del último inciso (d), el que abarca lo que llamamos trabajo infantil peligroso. Esta precisión es importante, diferenciándolo del campo del trabajo infantil en general, y del conjunto de las peores formas de trabajo infantil. Las otras tres formas (incisos $a, b, c)$ son llamadas en el mencionado Informe Global de la OIT como "formas incuestionablemente peores de trabajo infantil".

La definición general realizada en el Convenio 182 se amplía en la Recomendación 190 (1999). En esta Recomendación se establecen las actividades que por su naturaleza o por las condiciones en que se realizan implican mayores riesgos para la población infantil:

a) los trabajos en que el niño queda expuesto a abusos de orden físico, psicológico o sexual;

b) los trabajos que se realizan bajo tierra, bajo el agua, en alturas peligrosas o en espacios cerrados;

c) los trabajos que se realizan con maquinaria, equipos y herramientas peligrosos, o que conllevan la manipulación o el transporte manual de cargas pesadas;

d) Ios trabajos realizados en un medio insalubre en el que los niños estén expuestos, por ejemplo, a sustancias, agentes o procesos peligrosos, 0 bien a temperaturas o niveles de ruido o de vibraciones que sean perjudiciales para la salud, y

e) los trabajos que implican condiciones especialmente difíciles, como los horarios prolongados o nocturnos, o los trabajos que retienen injustificadamente al niño en los locales del empleador.

El artículo 4 del Convenio en referencia menciona el compromiso que asumen los países respecto al trabajo infantil peligroso:

1. Los tipos de trabajo a que se refiere el artículo 3, d) deberán ser determinados por la legislación nacional o por la autoridad competente, previa consulta con las organizaciones de em- 
pleadores y de trabajadores interesadas y tomando en consideración las normas internacionales en la materia, en particular los párrafos 3 y 4 de la Recomendación sobre las peores formas de trabajo infantil, 1999.

2. La autoridad competente, previa consulta con las organizaciones de empleadores y de trabajadores interesadas, deberá localizar dónde se practican los tipos de trabajo determinados a tenor del párrafo 1 de este artículo.

3. D eberá examinarse periódicamente y, en caso necesario, revisarse la lista de los tipos de trabajo determinados a tenor del párrafo 1 de este artículo, en consulta con las organizaciones de empleadores y de trabajadores interesadas.

\section{El rol de la seguridad y salud en el trabajo}

Desarrollando su rol de colaboración con los países, para la protección de los menores, IPEC-OIT ha empezado a sensibilizar y movilizar a socios no tradicionales como son las instituciones y especialistas del campo de la seguridad y salud en el trabajo, que tradicionalmente no han estado comprometidos en el proceso de identificación y priorización de formas de trabajo infantil peligroso, que permitan el retiro del niño de las labores de alto riesgo o el control de los riesgos cuando se trate de trabajo de menores entre 15 a 17 años.

H iba (2002), citando a Takala, indica que con frecuencia la reacción de los gobernantes ante un problema social como el del trabajo infantil peligroso, es de "medicarla". Sin embargo, los profesionales de seguridad y salud, si bien no pueden resolver los problemas sociales que originan el trabajo infantil peligroso, deben indicar el camino y mostrar qué debería hacerse desde el punto de vista de la promoción y la prevención. No es su responsabilidad exclusiva, pero pueden contribuir a resolver el problema, pueden coadyuvar a aliviar, asesorar y orientar, pero también de denunciar y alertar a la sociedad en materia de riesgos graves para la seguridad y salud de los niños que trabajan.

Constatamos que en América Latina existe una amplia experiencia en el campo de la seguridad y salud en el trabajo, que brinda una excelente oportunidad para establecer una alianza con las instituciones que están comprometidas con el tema del trabajo infantil. Este es el marco de la iniciativa planteada por IPEC-OIT para promover la creación de una red global sobre trabajo infantil peligroso que incorpore la perspectiva de la seguridad y salud en el trabajo.
La promoción de esta red se ha iniciado en Asia, África, América Central y América del Sur, en función a los siguientes objetivos:

- Apoyar a las Comisiones $\mathrm{N}$ acionales sobre trabajo infantil, a los programas nacionales y sub regionales del IPEC en el desarrollo de pautas y criterios por la identificación del trabajo infantil peligroso.

- Identificar y priorizar con mejores elementos técnicos especial izados en seguridad y salud, las formas más extremas de trabajo infantil peligroso en los países.

- Colaborar con visualizar y fundamentar las formas más peligrosas de trabajo infantil dentro de los proyectos existentes y en los planes de acción de los países.

- La preparación de materiales educativos sobretrabajo infantil peligroso.

- La identificación de acciones directas, por ejemplo investigaciones, evaluaciones, planes de mejoramiento del lugar de trabajo, soporte técnico entre instituciones, sobre formas de trabajo infantil peligroso.

- M ejorar las capacidades de intervención de las instituciones de seguridad y salud en el trabajo respecto al trabajo infantil peligroso, por ejemplo a través de información para compartir y elaborar una base de datos.

- Precisamente el rol que cumplen y pueden cumplir las instituciones y especialistas de la seguridad y salud en el trabajo cobra importancia en el campo del trabajo infantil peligroso. El conocimiento de estos sectores sobre la prevención de los factores de riesgo que pueden producir los accidentes de trabajo y las enfermedades ocupacionales, constituye una gran ayuda para las acciones en el campo de la prevención y tratamiento del trabajo infantil peligroso.

\section{Espacios de encuentros entre seguridad y salud y trabajo infantil peligroso en América del Sur}

En general, podemos identificar los siguientes niveles de encuentro en los que es posible establecer formas de colaboración entre la seguridad y salud en el trabajo y trabajo infantil peligroso. En sectores específicos:

- Los programas y proyectos que desarrollan las instituciones como el IPEC, por ejemplo en minería artesanal, agricultura, recolección de basura, construcción, ladrilleras. En estos programas y proyectos se ha avanzado de manera importante en el conocimiento de las condiciones de vida y trabajo del trabajador infantil en 
los sectores intervenidos, pero que no se ha agotado el aporte de la perspectiva de la seguridad y salud en el trabajo.

- Las investigaciones y actividades de promoción que diferentes instituciones del Estado, de la sociedad civil (universidades, ONGs) y otros, desarrollan en sectores similares, desde la perspectiva de la seguridad y salud en el trabajo.

A nivel de los países existen:

- Comisiones nacionales tripartitas sobre trabajo infantil, que tratan de promover la ratificación y aplicación de los Convenios 138 (Edad M ínima para el trabajo, de 1973), y particularmente del 182 (Las peores formas de trabajo infantil). Estas comisiones, 17 en total, tienen avances diferenciados, pero en general cuentan con planes nacionales de acción.

- Programas y proyectos de diversas instituciones, como el IPEC, que se proponen crear las condiciones para la eliminación de las peores formas de trabajo infantil.

- Instituciones de seguridad y salud en el trabajo de diferente tipo (del estado, universidades, ON Gs), que han realizado o están realizando acciones en el campo del trabajo infantil, sean investigaciones, actividades de promoción, educación, entre otras.

A nivel subregional tenemos:

- El proceso de integración de la Comunidad Andina, como es la discusión de una norma andina sobre seguridad y salud, el establecimiento de un observatorio laboral andino sobre progreso en materia de derechos laborales.

- El proceso de integración del M ercosur, a nivel de presidentes, en el Sub Grupo 10 sobre aspectos laborales y empleo, en la Comisión Parlamentaria, en el observatorio laboral que se está implementando.

- En estos espacios no está presente de manera adecuada el tema de trabajo infantil peligroso, menos aun desde la perspectiva de la seguridad y salud en el trabajo.

A nivel regional:

- La red sobre trabajo infantil que promueve el IPEC, que cuenta con más de dos mil miembros, una lista de intercambio de información, boletín electrónico, una página web interactiva. - La red de seguridad y salud en el trabajo (RSST), creada en 1999 y que cuenta con auspicio de OIT y OPS, de alcance global, y que cuenta con cerca de mil suscriptores que se comunican cotidianamente sobre temas de seguridad y salud en el trabajo.

- Espacios virtuales como la página web de la OIT sobre Trabajo sin riesgo, y la biblioteca vir- tual de la OPS. Se puede acceder a todos estos sitios desde <http://www.rsst.net>

\section{La Red sobreT rabajo Infantil Peligroso (Red TIP)}

La conformación de la Red TIP responde a la siguiente constatación: limitaciones conceptuales y metodológicas, magnitud del problema ( $48.5 \%)$, diversidad de sectores y actividades laborales, efectos a corto y largo plazo: "mucho que aprender", demanda de los países.

La Red TIP fue conformada en Reuniones Preparatorias en América del Centro y América del Sur, en marzo del 2002, promovidas por la OIT a través de su Programa Internacional para la Erradicación del Trabajo Infantil (IPEC), y tiene como finalidad fortalecer y apoyar a los países de la región, principalmente a sus Comités $\mathrm{N}$ acionales de Erradicación del Trabajo Infantil (conformados en el marco de los M emorandos de Entendimiento firmados entre el IPEC y las autoridades nacionales respectivas), para el desarrollo de actividades destinadas a eliminar el trabajo infantil.

En el caso de la Red TIP para América del Sur la reunión se llevó a cabo en Lima, Perú, del 20-22 de marzo de 2002, con el lema: "Construyendo alianzas para la identificación y priorización de las acciones sobre el trabajo infantil peligroso". Se invitaron a especialistas y representantes de organizaciones de empleadores, del Estado y de los trabajadores, con experiencia en trabajo infantil y en seguridad y salud en el trabajo de Argentina, Brasil, Colombia, Ecuador, Paraguay, Venezuela y Perú.

Teniendo como base el trabajo realizado por los grupos de expertos, en el que se identificaron productos a alcanzar a junio del 2003 (tomando como referencia el análisis de Fortalezas, O portunidades, D ebilidades, Amenazas), se formularon resultados y de los resultados se derivaron los objetivos estratégicos, la V isión y Misión de la Red TIP (Cuadro 5). También fue tomado en cuenta el debate sobre los aspectos conceptuales, los criterios para la identificación del TIP y las recomendaciones sobre las acciones a seguir.

La Visión de la Red TIP, formulada con los participantes de la reunión es: La Red TIP es un espacio de comunicación y articulación de instituciones, organizaciones y personas de A mérica Latina que realizan actividades relacionadas al trabajo infantil peligroso (TIP), contribuyendo a 
Cuadro 5

Objetivos estratégicos y resultados.

\begin{tabular}{|c|c|}
\hline Objetivos estratégicos & Resultados \\
\hline $\begin{array}{l}\text { 1. Promover la formulación y difusión } \\
\text { de herramientas especializadas en seguridad } \\
\text { y salud en el trabajo para la ETIP }\end{array}$ & $\begin{array}{l}\text { R1. Instituciones y organizaciones articuladas de manera flexible, abierta } \\
\text { y eficiente en una Red regional } \\
\text { R2. Experiencias sistematizadas y difundidas que aportan a la ETIP } \\
\text { R3. Protocolos de investigación e investigaciones en desarrollo. } \\
\text { R4. Criterios científico-técnicos establecidos para la determinación del TIP } \\
\text { de acuerdo al Convenio } 182 \\
\text { R5. Guías elaboradas y utilizadas para mejorar las condiciones de trabajo } \\
\text { y eliminar riesgos en el TIP de mayores de 14-15 años }\end{array}$ \\
\hline $\begin{array}{l}\text { 2. Contribuir a mejorar las capacidades } \\
\text { de los países para lograr avances normativos } \\
\text { a nivel nacional y subregional para la ETIP }\end{array}$ & $\begin{array}{l}\text { R6. Capacidades nacionales y subregionales mejoradas para lograr avances } \\
\text { legislativos para la ETIP } \\
\text { R7. Capacidades de las instituciones y organizaciones de la Red TIP fortalecidas } \\
\text { para el cabildeo y abogacía orientados a la ETIP }\end{array}$ \\
\hline $\begin{array}{l}\text { 3. Contribuir a mejorar las capacidades } \\
\text { institucionales y de recursos humanos } \\
\text { de los países para la ETIP }\end{array}$ & $\begin{array}{l}\text { R8. Capacidades nacionales mejoradas para articularse en redes de acción } \\
\text { para la ETIP } \\
\text { R9. Capacidades nacionales fortaleci das para establecer y desarrollar sistemas } \\
\text { de monitoreo e inspección para la ETIP }\end{array}$ \\
\hline
\end{tabular}

mejorar sus capacidades y sinergias para el desarrollo de actividades conjuntas a ni vel sectorial, nacional y regional, orientados a la eliminación del trabajo infantil peligroso.

La M isión de la Red TIP, identificada en la mencionada reunión es: La Red TIP en un conjunto de organizaciones e instituciones articuladas, con capacidad de incidencia política, solvencia científica y legitimidad social para promover y monitorear iniciativas de eliminación del Trabajo I nfantil Peligroso (ETIP)".

En la reunión se establecieron compromisos y recomendaciones por países y por líneas de acción. Se dieron las siguientes recomendaciones:

- Acercarse a las sociedades de pediatría.

- Conformar redes nacionales operativas.

- Articular la Red TIP a otras redes como la Red de Seguridad y Salud en el Trabajo.

- Alcanzar los compromisos a los Comités Nacionales.

\section{Los avances a la fecha}

Con diferente nivel de operatividad, se han establecido instancias de coordinación sobre TIP en los países: Brasil (se sostuvo una reunión en Salvador de Bahía), Colombia, Ecuador, Perú, Venezuela. Enlazando especialistas y responsables de trabajo infantil y de seguridad y salud en el trabajo.
Se ha realizado un taller técnico sobre "Criterios para la definición del Trabajo Infantil Peligroso/TIP", en agosto, en Quito, Ecuador (IPEC-OIT, 2002). Se reunieron especialistas con el objetivo de:

- Analizar y recuperar las propuestas existentes (estado del arte) sobre los criterios para definir trabajo infantil peligroso.

- Definir categorías y variables que permitan la operacionalización del trabajo infantil peligroso, bajo referentes que pueden ser estandarizados para los diferentes países de América Latina y del resto del mundo.

- Definir criterios para la identificación, categorización y priorización del TIP.

La reunión tuvo los siguientes momentos:

- La exposición y revisión de los Convenios de la OIT 138 y 182, como marco de referencia del trabajo del Taller.

- Los avances que a nivel global y de los países se conoce sobre los avances en la aplicación de los Convenios mencionados.

- El estado de avance para la definición de criterios sobre TIP, así como la conformación de la Red sobre Trabajo Infantil Peligroso (Red TIP).

Se debatió sobre la perspectiva más adecuada para abordar la definición de criterios sobre trabajo infantil peligroso: a) por actividades económicas; b) por factores de riesgo; y c) por efectos en la salud. 
Los asistentes concluyeron que cada una de estas perspectivas tiene aspectos que facilitan 0 dificultan la definición del TIP y su aplicación por los países.

La perspectiva de abordarlo por actividades económicas es más cercana a la necesidad de los países que deben establecer de manera técnica y factible de qué sectores deben de erradicarse el TIP. Finalmente es en actividades económicas reales donde se ubican los niños expuestos a estas formas de trabajo.

La perspectiva de abordar la definición del TIP por los efectos permite mostrar el impacto de éstas formas de trabajo en la salud y seguridad del menor y podría sensibilizar a las autoridades y a la opinión pública, incluso logrando ampliar el espectro de aliados en esta tarea.

La perspectiva de abordar la definición de TIP por factores de riesgo permite evitar que se dupliquen criterios para actividades diversas con riesgos similares; además puede facilitar la labor de los especialistas de seguridad y salud en el trabajo que ayudan a los países en esta tarea.

En la perceptiva de abordaje por actividades económicas, se pensó en algún momento utilizar la clasificación internacional uniforme de actividades económicas, pero se consideró que esta clasificación respondía a otras necesidades, no siendo muy útil para identificar la diversidad de procesos peligrosos, condiciones de trabajo y factores de riesgo en las actividades económicas, las mismas que dentro de un mismo sector tienen diferentes tipos de empresas por tamaño, tecnología y por tanto, coexisten diversos procesos productivos que no llegan a ser identificados por la clasificación mencionada.

Es cierto que finalmente los países deberán establecer de qué actividades económicas peligrosas se debe retirar el trabajo infantil. Pero a este taller le correspondía sugerir criterios de carácter y aplicación más general. Por ello, si bien la reunión consideró las 3 dimensiones 0 perspectivas de abordar el tema, se centró en las dos últimas.

Sin embargo, cual quiera que sea la perspectiva que se asuma se debe considerar las otras dimensiones. El Taller ha llegado a algunas conclusiones y sugerencias que pueden ser de utilidad para avanzar en la definición de criterios sobre TIP:

- Propuesta para lista de cero tolerancia, incluyendo la lista de factores de vulnerabilidad.

- Listado de criterios (a varios niveles).

- Propuesta sobre factores de riesgo.
- Propuesta para organizar una guía para los países para determinar el TIP.

Estas conclusiones fueron revisadas posteriormente, en otra reunión de expertos en Ginebra, en la que se realizaron aportes y que serán próximamente difundidos.

\section{Desarrollo de proyectos específicos}

Se ha establecido una red electrónica de la Red TIP, que cuenta a la fecha con unos 180 suscriptores y que tiene una actividad regular, si bien aún no tan intensa. Esta red permite intercambiar experiencia sobre intervenciones, investigaciones, actividades en relación al trabajo infantil peligroso. Se espera que progresivamente pueda tener una mayor actividad y que aporte al trabajo de los países.

Con la finalidad de poder contar con experiencias directas que puedan apoyar las experiencias de los países, I PEC-OIT apoyó pequeñas iniciativas que están culminando y seran difundidas próximamente:

En Ecuador, como se mencionó, se apoyó la realización de la Reunión de expertos para proponer criterios de definición del Trabajo Infantil Peligroso.

En Perú se han apoyado dos iniciativas:

- La sistematización de criterios utilizados en el trabajo infantil en la minería artesanal, para definir trabajo infantil peligroso, y que pueda ser aplicado a otros sectores. Pero además se está elaborando un instrumento para la calificación del nivel de peligro de las actividades.

- El diagnóstico de las condiciones de trabajo de los menores de 14 años cargadores carretilleros de mercado. Así como la valoración del nivel de riesgo y la sugerencia de algunas soluciones prácticas para su prevención en el trabajo de cargadores de carretillas de mercados de menores de 14 a 17 años.

En Brasil se ha apoyado el trabajo de sistematización de la experiencia del país en la erradicación del trabajo infantil, en lo concerniente a la investigación, formación e inspección, que luego debe servir, en la forma de un módulo autoinstructivo, para su difusión a otros países.

\section{Los avances en Centro-América}

Como parte de las actividades desarrolladas desde octubre del 2002 hasta abril del 2003, se tiene 
la realización de talleres de conformación y de consolidación de las redes nacionales contra el trabajo infantil peligroso, en cada uno de los países de Centro-América, República Dominicana y Panamá. Estos talleres permitieron organizar las redes nacionales conformadas por las organizaciones, instituciones públicas, privadas, organizaciones de trabajadores y empleadores, centros de estudios e investigación, asociaciones de profesionales en pediatría, medicina del trabajo, salud y seguridad en el trabajo etc.

Toda la información respecto a la Red TIP en Centro-América, República Dominicana y Panamá puede ser vista en <http://ipec.oit. or. $\mathrm{Cr} />$

La idea fundamental de las organizaciones e instituciones de la Red TIP es convertirse en el brazo técnico en material de salud y seguridad en el trabajo y en especial en lo relativo a trabajo infantil peligroso de los Comités Directivos $\mathrm{N}$ acionales de Erradicación del Trabajo Infantil a la luz de lo que establece el convenio 182 de la OIT.

Es importante además señalar que las diferentes actividades que se realizan en los países, como por ejemplo mapeo de peores formas, investigaciones sobre condiciones y medio ambiente del trabajo infantil, en fin todos aquellos trabajos relacionados con el quehacer de la Red TIP, son coordinadas con la red en cada país, de manera que haya mayor participación e involucramiento de sus miembros. Actualmente se trabaja en la elaboración de un protocolo para la realización de estudios sobre condiciones y medio ambiente del trabajo infantil en la agricultura.

\section{Conclusión}

Muchos de nosotros, sea por la necesidad económica, por motivos culturales o por tradición familiar, hemos debido de trabajar cuando menores, lamentablemente no todos tienen la suerte de superar esta situación sin afectar su integridad física, mental y moral. N os correspondea todos contribuir a que los niños del mundo no tengan que trabajar, sino estudiar y jugar, contribuir a superar esta situación de injusticia extrema, de menores que trabajan con riesgo para su salud, su seguridad e, incluso, su vida.

\section{El niño yuntero}

Miguel Hernández

(poeta español, de niño pastor)

Carne de yugo ha nacido más humillado que bello con el cuello perseguido por el yugo para el cuello.

Empieza a vivir y empieza a morir de punta a punta levantando la corteza de su madre con la yunta.

Contar sus años no sabe y ya sabe que el sudor es una corona grave de sal para el labrador.

Me dueleeste niño hambriento como una grandiosa espina y su vivir ceniciento revuelve mi alma de encina. 


\section{Referencias bbliográficas}

Alarcón W 2001. Trabajar y estudiar en los Andes. A proximación al trabajo infantil en las comunidades rurales de Cuzco y Cajamarca. Unicef, Lima.

Alcocer M \& Forastieri V 2003. Informe de actividades de la Red TIP en Centroamérica. OIT, San José.

Forastieri V 1997. Children at work: health and safety risks. OIT. Ginebra.

Hiba 2002. La seguridad y salud en el trabajo infantil peligroso. Ponencia presentada en la Reunión Preparatoria de la Red TIP. OIT, Lima.

INFA (Instituto Nacional del Niño y la Familia) 2001. Entre el barro y el juego. Proyecto de Erradicación del Trabajo Infantil en las ladrilleras del sur de Quito. Programa de protección y educación a niños y niñas que trabajan. IPEC-OIT, Quito.

IPEC-OIT 2001. N iños que trabajan en la minería artesanal de oro en el Perú. Estudio nacional sobre el trabajo infantil en la minería artesanal. OIT, Lima.

IPEC-OIT 2002. Criterios para la definición del Trabajo Infantil Peligroso. Documento de trabajo. Informe del
Taller Técnico de Quito, 7-9 de agosto de 2002 (documento en preparación para su edición)

OIT 1973. Convenio 138. Convenio sobre la edad mínima de admisión al empleo.

OIT 1999. Convenio 182. Convenio sobre la prohibición de las peores formas de trabajo infantil y la acción inmediata para su eliminación.

OIT 1999. Recomendación 190. Recomendación sobre la prohibición de las peores formas de trabajo infantil y la acción inmediata para su eliminación.

OIT 2002. Informe Global "Un futuro sin trabajo infantil". Informe del Director General de la OIT. Conferencia Internacional del Trabajo 90a Reunión.

Piedrahita H 2002. Algunas explicaciones sobre el mayor riesgo de los menores a accidentes y enfermedades ocupacionales. M onografía.

Artigo apresentado em 15/9/2003

Aprovado em 20/10/2003

Versão final apresentada em 10/11/2003 